\title{
COMPREENSÁO DE PACIENTES SOBRE A CONTINUIDADE DO PÓS- OPERATÓRIO, A PARTIR DO AUTOCUIDADO DOMICILIAR
}

\author{
PATIENT'S UNDERSTANDING OF POST-OPERATIVE CARE \\ CONTINUITY REGARDING HOME SELF-CARE
}

\section{COMPRENSIÓN DE LOS PACIENTES SOBRE LA CONTINUIDAD DEL CUIDADO POSTOPERATORIO, A PARTIR DEL AUTOCUIDADO DOMICILIAR}

\author{
Micaelle Oliveira De Luna Freire* \\ WiLliane LeÔNCIO GOMEs** \\ João Euclides Fernandes Braga*** \\ JaQueline QueIroz De MaCÊDo****
}

\begin{abstract}
RESUMO
Objetivo: Analisar a compreensão e os sentimentos de pacientes no pós-operatório sobre a continuidade do autocuidado domiciliar. Material e método: Estudo qualitativo, realizado com pacientes em pós-operatório, e condução de entrevista semiestruturada, submetida a análise temática a partir das Teorias de Autocuidado. Resultados: Participaram onze pacientes, internados na clínica cirúrgica. Emergiram duas categorias que abordam sentimentos relacionados à realização da cirurgia e fatores que influenciam a aprendizagem das medidas de autocuidado no pós-operatório. Tais achados evidenciam o objeto de trabalho da enfermagem junto à especialidade cirúrgica, e ressaltam a importância dos cuidados hospitalares educacionais e da continuidade pela atençáo primária. Conclusáo: Há interferência do estado emocional, apoio familiar e orientaçóes para alta nas medidas de autocuidado. Antes das medidas educativas, deve-se considerar o estado emocional do paciente e familiar de modo a favorecer a aprendizagem sobre o autocuidado.
\end{abstract}

Palavras-chave: Cirurgia; Alta do paciente; Ansiedade; Autocuidado.

\footnotetext{
*Enfermeira. Curso de Graduaçẫo em Enfermagem. Universidade Federal da Paraíba, João Pessoa, Brasil. ORCID: https://orcid. org/0000-0002-4847-0699 Email: mica_mcario@hotmail.com

**Enfermeira. Curso de Graduaçáo em Enfermagem. Universidade Federal da Paraíba, João Pessoa, Brasil. ORCID: https://orcid. org/0000-0003-0538-8537 Email: gomeswilliane.l@gmail.com

***Enfermeiro. Doutor em Farmacologia. Diretor do Centro e Ciências da Saúde e Professor do Departamento de Enfermagem em Saúde Coletiva. Universidade Federal da Paraíba, Joáo Pessoa, Brasil. ORCID: https://orcid.org/0000-0003-3413-6072 Email: joeufebra@gmail.com

****Enfermeira. Doutora em Ciências da Saúde. Professora do Departamento de Enfermagem Clínica. Universidade Federal da Paraíba, Joáo Pessoa, Brasil. ORCID: https://orcid.org/0000-0002-1330-3460 Email: jaquelineqmac@gmail.com. Autor correspondente.
} 


\begin{abstract}
Objective: To analyze the understanding level and the feelings of post-operative patients about the continuity of home self-care. Materials and methods: Qualitative study, carried out with eleven post-operative patients, using semi-structured interviews, subjected to thematic analysis based on Self-Care Theories. Results: Eleven patients, hospitalized in a surgical clinic, participated. Two categories emerged, addressing the feelings related to the surgery and the factors that influence the learning of self-care measures in the post-operative period. They underline the scope of nursing work, together with the surgical specialty; and emphasize the importance of educational hospital care and continuity in primary care. Conclusion: These findings suggest an interference of the emotional condition, family support and discharge guidelines for home self-care. Before taking any educational measures, it is necessary to consider the emotional state of the patient and family in order to increase self-care learning.
\end{abstract}

Key words: Surgery; Patient Discharge; Anxiety; Self-care.

\title{
RESUMEN
}

Objetivo: Analizar la comprensión y los sentimientos de los pacientes en el postoperatorio, sobre la continuidad del autocuidado domiciliar. Material y método: Estudio cualitativo, realizado con pacientes en postoperatorio, conducción de entrevista semiestructurada, sometida a análisis temático a partir de las Teorías de Autocuidado. Resultados: Participaron once pacientes internados en una clínica quirúrgica. Emergieron dos categorías que abordan los sentimientos relacionados con la realización de la cirugía y factores que influencian el aprendizaje de las medidas de autocuidado en el postoperatorio. Ellas evidencian el objeto de trabajo de la enfermería junto a la especialidad quirúrgica, y resaltan la importancia de los cuidados hospitalarios educativos y de la continuidad a través de la atención primaria. Conclusiones: Los hallazgos sugieren una interferencia del estado emocional, apoyo familiar y orientaciones de alta en las medidas de autocuidado en el domicilio. Antes de las medidas educativas, hay que considerar el estado emocional del paciente y familiar para favorecer el aprendizaje sobre el autocuidado.

Palabras clave: Cirugía; Alta del paciente; Ansiedad; Autocuidado.

Data de recepçáo: 13/11/2018

\section{INTRODUÇÁO}

O processo de hospitalização, em si, pode provocar sentimentos de ansiedade, e em pacientes cirúrgicos, assume uma situação ainda mais crítica, com o aparecimento de insegurança relacionada ao período perioperatório ${ }^{(1-3)}$. Alguns estudos evidenciam que a presença de sentimentos descontáveis, ansiedade e depressão na fase préoperatória pode gerar consequências no pósoperatório, como complicaçóes, adiamento da alta hospitalar e reinternaçôes constantes ${ }^{(4)}$.

A alta hospitalar é definida como uma transição da assistência ao paciente do hospital para outros serviços de saúde ou para o domicílio ${ }^{(5,6)}$ e decorre
Data de aceitação: 17/12/2019

de um planejamento do cuidado para o retorno à comunidade, que inclui o processo educativo com informaçóes para o paciente. Quando se refere a pacientes no pós-operatório, se torna imprescindível fornecer orientaçóes, com o intuito de favorecer a continuidade do cuidado no domicílio e a redução de desconfortos e complicaçôes ${ }^{(7,8)}$. Para um retorno seguro à comunidade, o fornecimento de orientaçóes de alta decorre da necessidade de autocuidado no processo de recuperação da saúde, o que requer conhecimento e suporte emocional e, a depender, certo domínio sobre as atividades a serem realizadas ${ }^{(9)}$.

Por autocuidado entende-se as capacidades de um indivíduo para desempenhar cuidados sobre 
si mesmos e o ambiente, de acordo com suas necessidades, com o intuito de promover benefícios, saúde e bem-estar para sua vida ${ }^{(10)}$.

Dentre os profissionais da equipe de saúde, a literatura indica que cabe ao enfermeiro a função de investigar e atuar diante desses sentimentos demonstrados pelos pacientes cirurgiados, através de intervençóes de enfermagem voltadas para redução da ansiedade desses indivíduos ${ }^{(11-13)}$. Assim, conforme as Teorias de Autocuidado de Orem, o enfermeiro tem como uma de suas atribuiçóes, o papel de desenvolver açôes que promovam autonomia para os indivíduos e familiares, dentre as quais por meio de estratégias de ensino ${ }^{(10)}$.

A Teoria Geral do Autocuidado é composta por três bases teóricas: a Teoria do Autocuidado, Teoria do Déficit do Autocuidado e a Teoria dos Sistemas de Enfermagem. Essas teorias estão relacionadas e articuladas entre si, e descrevem a prática do autocuidado pelos pacientes, o nível de demanda de autocuidado, as intervençóes de enfermagem respectivamente. A Teoria do Déficit do Autocuidado manifesta a incapacidade do indivíduo em realizar os cuidados necessários para sua saúde e reflete a importância do papel da enfermagem diante dessas dificuldades. Essa condição ressalta a Teoria dos Sistemas de Enfermagem que representa as açôes que o enfermeiro pode desenvolver através de orientaçóes sobre o autocuidado, que promovam autonomia para os indivíduos e familiares, dentre as quais as estratégias de ensino ${ }^{(10)}$.

Nesse sentido, os pacientes submetidos a um procedimento cirúrgico necessitam de uma educação em saúde adequada e abrangente, devido à redução do tempo de hospitalização após a cirurgia $^{(14)}$, além de possibilitar um empoderamento ao paciente em relação aos cuidados necessários com sua saúde ${ }^{(15)}$. Dessa forma, compreende-se a importância de atividades educativas para pacientes no pós-operatório, levando em consideração os fatores que podem comprometer a percepção desses indivíduos diante do estado vulnerável provocado por uma cirurgia ${ }^{(16)}$.

Diante da necessidade de autonomia e segurança para continuidade do tratamento após a alta hospitalar, entre pacientes com características de ansiedade, este estudo se justifica por responder ao seguinte objetivo: analisar a compreensão e os sentimentos de pacientes no pós-operatório quanto à continuidade do autocuidado domiciliar.

\section{MATERIAL E MÉTODO}

Trata-se de uma pesquisa descritiva, com abordagem qualitativa $^{(17)}$ realizada com onze pacientes no pósoperatório internados na clínica cirúrgica, com possível estado de ansiedade, de um hospital escola federal localizado no Nordeste do Brasil. Para isso, foi realizada entrevista com os pacientes, aplicação da Escala Hospitalar de Ansiedade e Depressão ${ }^{(18)}$ para os pacientes que concordaram em participar do estudo, e apresentaram grau de ansiedade acima de oito. A amostra da pesquisa foi determinada por critério de conveniência até o limite de saturação dos dados. Esses participantes corresponderam aos seguintes critérios de elegibilidade: maiores de 18 anos; internados na Clínica Cirúrgica e oriundos de procedimentos cirúrgicos; com capacidade física e mental de responderem ao estudo; com grau de ansiedade acima de oito, que representa possível estado de ansiedade. Essa escala ${ }^{(18)}$ possui 14 itens, dentre estes, sete são voltados para a avaliação da ansiedade e sete para a depressão. Para o presente estudo, foi realizada a avaliaçáo apenas da ansiedade. Foram adotados os pontos de cortes, conforme estudo $^{(18)}$, que consistem em: sem ansiedade de 0 a 8 , com ansiedade $\geq 9$. A escolha dessa escala deve-se a facilidade de aplicaçáo e a possibilidade de identificar sintomas de ansiedade em pacientes internados em hospitais gerais. Foram entrevistados 25 pacientes, dentre estes, 11 apresentaram grau de ansiedade igual ou maior que nove, de modo que 11 compuseram a amostra final.

A coleta de dados foi realizada pela pesquisadora durante o período de julho a setembro/2018 através de levantamento de dados nos prontuários e entrevista semiestruturada. A entrevista foi realizada pela primeira pesquisadora, gravada em mídia digital, em local reservado da clínica cirúrgica, com média de duração de aproximadamente 20 min. Após o consentimento, os participantes respondiam ao formulário de caracterização, a Escala Hospitalar de Ansiedade e Depressão e ao roteiro semiestruturado de entrevista.

A análise dos dados foi realizada por meio da análise temática ${ }^{(19)}$, como segue: 1) transcrição, leitura e listagem de assuntos abordados; 2) organização de códigos; 3) agrupamento dos códigos em temas; 4) recodificaçáo do conjunto de dados em um processo contínuo de análise do estudo; 5) estabelecimento das temáticas e interpretação à luz 
das Teorias de Autocuidado de Orem ${ }^{(10)}$.

Esta pesquisa seguiu as regulamentaçóes da Declaração de Helsinki de 1964 e da Resolução do Conselho Nacional de Saúde do Brasil no 466/2012. Assim, o estudo obteve prévia aprovação do Comitê de Ética em Pesquisa (CAAE no 86689318.5.0000.5183) e esclareceu aos participantes sobre a pesquisa e as normas éticas, recebendo autorização com a assinatura do Termo de Consentimento Livre e Esclarecido. Como forma de garantir o anonimato, os relatos foram identificados pelo número da entrevista do participante.

\section{RESULTADOS}

Participaram deste estudo onze pacientes que encontravam-se no pós-operatório mediato de procedimentos cirúrgicos devido a neoplasia gástrica (1), cálculos renais (1), miomatose uterina (6), cálculos na vesícula biliar (2) e câncer de mama (1). Tais sujeitos têm idade entre 30 a 70 anos, com predomínio de pessoas do sexo feminino (90,9\%), e escore de ansiedade com média de 11 , o que significa possível estado de ansiedade.

Foram evidenciadas duas categorias que relacionam o estado emocional, e fatores de ordem educativa e familiar quanto à continuidade do autocuidado no pós-operatório após a alta hospitalar: Sentimentos relacionados à realização da cirurgia e Fatores extrínsecos que influenciam a aprendizagem das medidas de autocuidado.

\section{CATEGORIA A. Sentimentos relacionados à cirurgia que interferem no autocuidado}

A insegurança quanto à recuperaçáo e realização do autocuidado deve-se a questóes relativas à dificuldade na realização das atividades de vida diária, cuidado com a família, atividades domésticas, e o próprio autocuidado. Quanto a isso evidencia-se o fato de se tratarem, predominantemente, de mulheres que assumem o cuidado para com a família.

[Preocupada acerca] De fazer a comida, lavar roupa, que os meus meninos náo faz isso. E fazer a comida, ir comprar alimentação também. Essas coisas que eu vou sentir dificuldade [...]. Eu já fico tensa, preocupada, ansiosa, nervosa, porque não vou ter como fazer as coisas direito (P-4).
Eu me sinto preocupada, porque eu faço tudo assim né? Mas eu tô ciente que náo posso, porque tem um tempo indicado pra isso né? Vou ter que encarar isso [...] (P-7).

Porém, a insegurança também se deve ao receio com a realização do autocuidado requerido pela cirurgia.

[...] Eu tenho que ser forte, e fazer do mesmo jeito aqui do hospital né? Me cuidar, pra náo pegar infecção né? E ficar boa né? Terminar o tratamento, fazer o máximo (P-4).

Ah, eu me sinto assim, preocupada, porque no hospital é uma coisa em casa é outra né? É isso [...]. Muito triste [...]. Porque eu... (choro) (P-11).

Por outro lado, a realização do procedimento cirúrgico ocasiona uma ambiguidade de sensaçóes, com alívio devido à percepção de resolução do problema de saúde e sensação de tensão devido aos cuidados requeridos.

Eu me sinto... vou fazer nada né? Porque se eu for fazer, para piorar né, e depois voltar de novo [...]. Tồ me sentindo bem né? [...]. Eu vivia meia preocupada [...]. Eu vou ficar tranquila (P-8).

Uma fonte de apoio que favorece a sensação de alívio/segurança relatado pelos participantes relaciona-se ao suporte da espiritualidade, através do qual expóem o agradecimento pela resolução do problema.

Tô bem, graças a Deus [...]. Com certeza, primeiramente Deus, segundo minha filha, que tá ai pra me ajudar, [...]. Até recuperar, porque não dá pra eu ficar sozinha [...] (P-10).

Eu tô me sentindo bem, no sentido de que eu tô dando graças a Deus [...]eu graças a Deus tô bem, não tô sentindo dores diferenciadas, só a dor mesmo da cirurgia né, do procedimento. [...]. A gente acredita que vai ficar tudo bem, mas sempre tem um temorzinho (P-9).

\section{CATEGORIA B. Fatores extrínsecos que influenciam a aprendizagem das medidas de autocuidado}

No que se refere à percepçáo sobre o autocuidado no pós-operatório, os participantes referem aspectos quanto às orientaçóes para alta hospitalar recebidas, e o suporte necessário para o autocuidado.

Com relação às estratégias educativas para 
alta hospitalar, quando os participantes relatam o recebimento de explicaçóes sobre a continuidade do cuidado, estas são provenientes da fonte da equipe de enfermagem, revelando o papel de educador praticado por esta profissão.

É mais o enfermeiro mesmo, que orienta mais assim. É mais a parte de enfermagem que passa aqui orientando, ter cuidado... A enfermeira quando veio ela explicou direitinho, tirou o curativo explicou que eu tenho que tá secando, explicou que eu tenho que secar de um lado só pra o outro, e náo ficar esfregando, a importância de ficar bem sequinha. Então, explicaram bem direitinho (P-3).

Diante dos relatos, a atuação do enfermeiro foi ressaltada no que diz respeito as orientaçóes fornecidas para o autocuidado. Sendo o enfermeiro um profissional que estabelece vínculo maior com o paciente, nesse momento de orientaçóes para alta hospitalar se faz necessário sanar as dúvidas e anseios de cada indivíduo, que é possível através da educação em saúde. Essa atribuição do enfermeiro como educador permite proporcionar mais segurança e autonomia aos pacientes e familiares na realização dos cuidados no domicílio.

Todavia, a ausência de explicaçóes por parte dos profissionais do serviço foi predominantemente relatada pelos participantes.

Ainda não [recebi orientação], né? Pelo fato de eu ainda tá por aqui, acho que no final eles vão dizer alguma coisa sobre isso (P-1).

[...] Não, eles não falaram nada não em relação dos cuidados não, médico só falou pra mim vir, daqui a 15 dias pra tirar os pontos, pegar o laudo da biopsia, e pegar a consulta do retorno da parte ginecológica (P-6).

[...] As pessoas de fora tudo me falam, mas do médico mesmo que é para gente ouvir[mexeu a cabeça que não] (P-7).

Importante evidenciar, que muitas das orientaçóes recebidas pelos participantes decorrem de acadêmicas de Enfermagem, participantes do projeto de extensão Orientação para alta de pacientes submetidos a procedimentos cirúrgicos. Tais relatos evidenciam a importância da realização de açóes de extensão, que dentre vários objetivos fornece um retorno da universidade para a comunidade.

Pois é, [...] a menina estava até conversando comigo sobre isso hoje (as meninas do projeto), porque assim, tem que ter o cuidado né? Nâo é só a cirurgia aqui. [...] E como que eu penso pra me cuidar? Ah, eu vou aproveitar e vou repousar. Lógico né, tem essa parte do repouso, mas é o se cuidar em si, assim, com a alimentação, ter cuidado para não fazer nenhum esforço físico. E ter o maior cuidado com isso, uma vez que a gente sabe que isso ai é o que vai me ajudar na minha recuperação (P-3).

Tais relatos demonstram que as açôes de educação em saúde podem contribuir positivamente com o equilíbrio emocional. Esse benefício reflete na sensação de segurança para os pacientes, e consequentemente na continuidade do cuidado após a alta hospitalar.

Foi. Ela [extensionista] disse pra eu não me baixar mais como me baixava, tem que ser na forma agachando. E não fazer esforço durante um periodo de mais ou menos 1 mês, porque quando eu trabalho de auxiliar de cozinha assim, é muito pesado (P-6).

Ao exporem as informaçóes que receberam através das açóes de educação em saúde, percebe-se que muitas das orientaçóes foram captadas de modo superficial, o que pode decorrer do estado emocional de ansiedade que os participantes se encontravam. Contudo, questiona-se se sem dessas açóes de ensino, o nível de ansiedade desses participantes poderia se encontrar ainda mais elevado, visto que a compreensão sobre o autocuidado influencia a sensação de autonomia após a alta hospitalar.

Outro fator relatado que interfere diretamente sobre o autocuidado trata-se da necessidade de um suporte familiar e social para uma recuperação efetiva, assim como a importância de uma rede de atenção básica que propicie o cuidado do paciente e seus familiares através da atenção domiciliar.

Eu ainda tô insegura, mas eu acho que vou pra casa de mamãe sabe, pedir ajuda a ela [...] (P-4).

[...] A ajuda que vou ter pra continuar o tratamento é dele (marido) [...]. E tem a enfermeira do posto [...] (P-11).

Mas eu assim, ela [irmã que veio ajudar] faz alguma coisa, e quando minha irmá for vou ver o que faço né? Providenciar uma pessoa para estar me ajudando (P-7). 
Dentre os fatores que interferem na prática do autocuidado, a família é um dos aspectos centrais que pode contribuir favoravelmente, aumentando a sensação de segurança e autonomia da pessoa em pós-operatório. Por outro lado, na ausência dessa compreensão por parte da família, o autocuidado fica comprometido.

Eu voi ficar tranquila. Não vou ficar aperriada, porque náo tenho menino novo, só filho mais velho em casa, que me aperreiam bastante [...] É, daqui eu vou pra casa das minhas meninas. Vou ficar em casa não [...] por que meu novo aperreia, que bebe. (P-8).

A partir da exposição dos relatos, observamse as dificuldades decorrentes da falta do suporte familiar após a alta hospitalar. Essa condição pode prejudicar o processo de recuperaçáo do paciente, por interferir nos aspectos físicos e emocionais que envolvem uma pessoa no pós-operatório, podendo comprometer a continuidade do autocuidado. Por isso, ressalta-se a importância das açóes de educação em saúde abrangerem também o familiar, de modo que a pessoa cirurgiada possa cuidar-se nesse momento vulnerável.

\section{DISCUSSÁO}

No presente estudo, o sentimento de insegurança para prática do autocuidado após a alta hospitalar foi um dos mais relatados pelos participantes, o que pode ser diretamente relacionado ao grau de ansiedade dos mesmos. Diante de um procedimento cirúrgico, as pessoas se tornam mais fragilizadas emocionalmente, por vivenciar um risco para sua saúde, que pode configurar um desafio no pósoperatório. Assim, sentimento de tensão durante a recuperação no pós-operatório e dificuldade em restabelecer sua autonomia foram apontados em estudo ${ }^{(20)}$, bem como alteraçóes no âmbito emocional, como presença de ansiedade severa a moderada, preocupação, baixo astral, solidão e abandono também foram identificadas mesmo após um mês ocorrência de cirurgias ortopédicas e gerais $^{(21)}$.

A vulnerabilidade emocional pode acometer a prática do autocuidado no domicílio, e prejudicar o processo de recuperaçáo do paciente, como exposto nas Teorias de Orem ${ }^{(10,20)}$. Essa incapacidade inclui atividades diárias básicas como realizar a higiene pessoal, que pode se tornar um obstáculo para pacientes no pós-operatório.

A assistência da equipe envolvida deve ser voltada tanto para os cuidados físicos, quanto emocionais. Tais açóes devem estar inseridas no planejamento de alta do paciente, favorecendo o entendimento desses sujeitos, e assim, assegurando a continuidade dos cuidados no domicílio ${ }^{(22,23)}$.

Os participantes relataram o recebimento de explicaçóes sobre a continuidade do cuidado provenientes da equipe de enfermagem, reforçando o papel dessa profissão exposto na Teoria Geral do Autocuidado, especificamente na Teoria dos Sistemas de Enfermagem que expóe a educação em saúde como uma das medidas de promoção do autocuidado ${ }^{(10)}$. Ademais, cabe a qualquer profissional de saúde a responsabilidade pela educação em saúde, partindo do pressuposto de um modelo de atenção à saúde baseado na prática do autocuidado $^{(24,25)}$.

Os relatos também destacaram o suporte de espiritualidade, que representou uma sensação de alívio pela resolução do problema de saúde. Proporcionar açóes voltadas para uma atenção espiritual no ambiente hospitalar, promove apoio e suporte para o paciente e familiares no processo de recuperação da saúde ${ }^{(26)}$.

Os pacientes podem voltar para suas residências com curativos, sondas, drenos, e dentre outros dispositivos que este e seus familiares desconhecem, criando assim, estresse e angústia pela ausência de instrução dos cuidados necessários em tais condiçôes ${ }^{(27,28)}$. A procura pela atenção primária foi relatada como uma alternativa para a continuidade do autocuidado, ressaltando a importância da atenção básica, e da atuação do enfermeiro na continuidade dos cuidados, bem como da necessidade de referência e contrarreferência entre os serviços de saúde ${ }^{(29-32)}$. Pois enquanto hospitalizado há o suporte do serviço, enquanto que no domicilio os pacientes relatam apreensão no autocuidado.

Utilizar o processo de enfermagem como ferramenta para a construção da aprendizagem do paciente sobre o autocuidado, favorece a identificação do tipo de atenção que este necessita, pois conforme a Teoria dos Sistemas de Enfermagem o sujeito pode ser classificado conforme seu grau de autonomia, quanto a incapacidade para realizar o autocuidado devido as suas limitaçóes; ou realizar os 
cuidados com ajuda; ou ser agente do autocuidado, possibilitado pela atenção fornecida previamente pelo enfermeiro ${ }^{(10)}$.

Por outro lado, a ausência de orientaçôes de alta por parte dos profissionais do serviço foi relatada pela maioria dos participantes do estudo. As orientações recebidas, em geral, decorrem de projeto de extensão de orientação de alta hospitalar. Esses achados ressaltam a importância da realização de açôes de extensão, que contribuem minimizando deficiências e melhorando os serviços de saúde ${ }^{(33)}$. Esses benefícios fornecidos pela extensão universitária contribuem para o atendimento de demandas sociais, através de mudanças significativas no conhecimento do paciente.

Os participantes expóem certa sensação de segurança obtida a partir dessas açóes de educação em saúde. Todavia, percebe-se que houve perda de informaçốes da ação educativa realizada, que pode se relacionar ao estado emocional que os participantes se encontravam. Sobre isso, a literatura expóe que a ansiedade interfere na apropriação cognitiva das informaçóes, dificultando a compreensão das mensagens ${ }^{(34,35)}$. Por tal motivo, faz-se importante que o profissional de saúde tenha atenção ao estado emocional do paciente, no momento de realizar orientaçôes, buscando acalmá-lo se necessário, e verificar o nível de compreensão do sujeito após o fornecimento das orientaçóes.

Diante disso, o processo de alta hospitalar inclui sanar todas as dúvidas do paciente e investigar se a mensagem foi compreendida pelo indivíduo e seus familiares ${ }^{(28)}$, fato reforçado pela Teoria do Autocuidado(10), que expóe a importância da identificação dos conhecimentos, habilidades, limitaçóes e motivaçóes dos pacientes para o planejamento das intervençóes.

Essas condutas podem ser realizadas através de orientaçóes verbais ou escritas, o que facilita a formação de vínculo entre o profissionalpaciente, com a finalidade de melhorar ansiedade e tensão apresentadas diante do procedimento cirúrgico ${ }^{(36-38)}$. Além disso, devem ser fornecidas conforme o contexto social, econômico e cultural do paciente, através de informaçôes que priorizem a qualidade e náo quantidade de orientaçóes ${ }^{(28,}$ 39). Por isso, o fornecimento de informaçôes para os pacientes confere uma conduta essencial, pois a partir do conhecimento sobre sua saúde, procedimento e tratamento realizado, o indivíduo adquire segurança, proporcionando redução da ansiedade e melhora na reabilitaçáo do quadro de saúde ${ }^{(2)}$.

Segundo a Teoria de Orem ${ }^{(10)}$ o suporte familiar constitui como um dos elementos dentre as unidades multipessoais, que envolve família, grupos e comunidades, como um fator relevante para o desenvolvimento do autocuidado. Além disso, considera-se o autocuidado como uma elaboração social, por receber interferência da convivência do meio social e familiar, somado aos fatores como idade, capacidade mental, convivência cultural e o próprio estado emocional( ${ }^{(24)}$.

Assim, um dos fatores contribuintes para um autocuidado efetivo consiste na participação das famílias e cuidadores dos pacientes, que contribui para reabilitação da saúde, visto que a família e o cuidador dos pacientes, muitas vezes assumem os cuidados domiciliares após a alta hospitalar ${ }^{(40)}$. Depreende-se que o suporte familiar contribui com o processo de recuperação, por possibilitar um auxílio nos cuidados realizados no domicílio, além do apoio emocional fornecido pelo ente familiar ou cuidador.

\section{CONCLUSÓES}

A análise dos resultados evidenciou sentimentos como a insegurança sobre a recuperação e ambiguidade de sensaçôes; além de fatores extrínsecos que influenciam a aprendizagem das medidas de autocuidado, ressaltando a importância das orientaçóes para alta hospitalar e do suporte familiar e social na sensação de segurança para o desempenho do autocuidado no domicílio.

Conforme as teorias do autocuidado, sugerese a interferência do estado emocional, apoio familiar e orientaçóes para alta nas medidas de autocuidado. A educação e o apoio dos pacientes na continuidade do seu autocuidado contribuem de modo positivo na recuperação do indivíduo no pós-operatório. Para tanto, há a necessidade de a Enfermagem atentar às singularidades, uma vez que se percebeu uma relação entre a compreensão das orientaçóes, o estado emocional e a continuidade do autocuidado domiciliar. Nesse momento, se faz necessário atenção ao estado emocional dos sujeitos, de modo que as explicaçóes fornecidas aos pacientes e familiares possam ser melhor 
compreendidas, e assim promovam a redução de ansiedade, insegurança e dúvidas desses indivíduos, assegurando o autocuidado após a alta hospitalar.

Como limitação deste estudo elenca-se o número de participantes, que se justifica pela metodologia e os critérios de inclusão. Todavia, tal quantidade não influenciou na qualidade dos resultados obtidos.

\section{REFERÊNCIAS}

1. Aliche JC, Ifeagwazi CM, Chukwuorji JC, Eze JE. Roles of Religious Commitment, Emotion Regulation and Social Support in Preoperative Anxiety. J Relig Health [Internet]. 2018 [cited 2018 may 09]; 59(2): 905-919. Available from: https:// www.ncbi.nlm.nih.gov/pubmed/30145628.

2. Coppetti L de C, Stumm EMF, Benetti ERR. Consideraçóes de pacientes no perioperatório de cirurgia cardíaca referentes às orientaçóes recebidas do enfermeiro. Reme: Rev. Min. Enferm [Internet]. 2015 [citado 2018 mai 16]; 19(1):11326. Disponível em: http://www.reme.org.br/artigo/ detalhes/990

3. Kazitani BS, Furuya RK, Dantas RAS, Dessotte CAM. Ansiedade e depressão pré-operatória: diferenças entre pacientes submetidos à primeira cirurgia cardíaca. Rev Rene [Internet]. 2018 [citado 2018 mai 19]; 19:e3079. Disponível em: https://pesquisa.bvsalud.org/enfermeria/resource/ pt/biblio-883201

4. Poole L, Leigh E, Kidd T, Ronaldson A, Jahangiri M, Steptoe A. The combined association of depression and socioeconomic status with length of post-operative hospital stay following coronary artery bypass graft surgery: Data from a prospective cohort study. J Psychosom Res [Internet]. 2014 [cited 2018 may 20]; 76(1):34-40. Available from: https://www.ncbi.nlm.nih.gov/pmc/articles/ PMC3991423/

5. Madigan SM, Fleming P, Wright ME, Stevenson M, Macauley D. A cluster randomised controlled trial of a nutrition education intervention in the community. J Hum Nutr Diet [Internet]. 2014 [cited 2018 jun 28]; 27 Suppl 2:12-20. Available from: https://www.ncbi.nlm.nih.gov/ pubmed/23656441

6. Weiss ME, Bobay KL, Bahr SJ, Costa L, Hughes RG, Holland DE. A Model for Hospital Discharge Preparation: From Case Management to Care Transition. J Nurs Adm [Internet]. 2015 [cited 2018 jun 17]; 45(12):606-14. Available from: http://europepmc.org/article/MED/26502068

7. Marques L de FG, Romano-Lieber NS. Estratégias para a segurança do paciente no processo de uso de medicamentos após alta hospitalar. Physis [Internet]. 2014 [citado 2018 jun 20]; 24(2):40120. Disponível em: http://www.scielo.br/pdf/ physis/v24n2/0103-7331-physis-24-02-00401.pdf

8. Mata LRF da, Carvalho EC de, Gomes CRG, Silva AC da, Pereira M da G. Postoperative selfefficacy and psychological morbidity in radical prostatectomy. Rev Lat Am Enfermagem [Internet]. 2015 [cited 2018 jun 15]; 23(5):806-13. Available from: https://www.scielo.br/scielo.php?pid=S0104$11692015000500806 \&$ script=sci_arttext $\&$ tlng=en

9. Prado Solar LA, González Reguera M, Paz Gómez N, Romero Borges K. La teoría Déficit de autocuidado: Dorothea Orem punto de partida para calidad en la atención. Rev Med Electron [Internet]. 2014 [citado 10 jul 2018]; 36(6):83545. Disponible en: https://www.medigraphic.com/ cgi-bin/new/resumen.cgi?IDARTICULO=53881

10. Orem DE. Nursing; concepts of practice. 5 th ed. St. Louis: Mosby, 1995. 478 p.

11. Gonçalves KKN, Da Silva JI, Gomes ET, Pinheiro LL de S, Figueiredo TR, Bezerra SMM da S. Anxiety in the preoperative period of heart surgery. Rev Bras Enferm [Internet]. 2016 [cited 2018 aug 10]; 69(2):397-403. Available from: http://www.scielo.br/scielo.php?pid=S0034$71672016000200397 \&$ script $=$ sci_arttext\&tlng=en

12. Inácio LA, Montezeli JH, Sade PMC, Caveiâo C, Hey AP. Atuação do enfermeiro nas orientaçôes de alta ao paciente pós-transplante renal. Rev Enferm UFSM [Internet]. 2014 [citado 2018 jun 18]; 4(2):323-31. Disponível em: https://pesquisa. bvsalud.org/portal/resource/pt/bde-27247

13. Paes DCZ, Lacerda MR, Hermann AP, Gomes IM, do Nascimento JD, Rodrigues JAP. Sugestôes de orientaçôes para alta de crianças no pós-transplante de células-tronco hematopoiéticas. Cogitare Enferm [Internet]. 2017 [citado 2018 jun 18]; 22(4):e50265. Disponível em: https://revistas.ufpr. $\mathrm{br} /$ cogitare/article/view/50265

14. OECD. Health at a Glance: Europe 2014 [Internet]. Paris: OECD Publishing; 2014 [cited 2018 jun 20]. 142 p. Available from: http://dx.doi. org/10.1787/health_glance_eur-2014-en

15. European Patients Forum (EPF). The European Patients' Forum Annual Work Plan 2015 [Internet]. Bruselas: EPF; 2015 [cited 2018 jun 20]. Available from: https://www.eu-patient.eu/globalassets/ library/annual-work-plan/epf-2015-annual-workplan.pdf

16. Ingadottir $\mathrm{B}$, Blondal $\mathrm{K}$, Jaarsma $\mathrm{T}$, Thylen $\mathrm{I}$. Perceptions about traditional and novel methods to learn about postoperative pain management: a qualitative study. J Adv Nurs [Internet]. 2016 [cited 
2018 jun 12]; 72(11):2672-83. Available from: https://onlinelibrary.wiley.com/doi/abs/10.1111/ jan. 13021

17. Bourgeault I, Dingwall R, Vries R. The SAGE Handbook of Qualitative Methods in Health Research. London: SAGE Publications Ltda; 2013. $786 \mathrm{p}$.

18. Zigmond AS, Snaith RP. The Hospital Anxiety and Depression Scale. Acta Psychiatr Scand [Internet]. 1983 [cited 2018 jun 05]; 67(6):36170. Available from: https://onlinelibrary.wiley. com/doi/abs/10.1111/j.1600-0447.1983. tb09716.x?sid=nlm\%3Apubmed

19. Braun V, Clarke V. Using thematic analysis in psychology. Qual Res Psychol [Internet]. 2006 [cited 2018 jun 05]; 3(2):77-101. Available from: https://www.tandfonline.com/doi/abs/10.1191/ 1478088706qp063oa

20. Forsberg A, Söderberg S, Engström Å. People's experiences of suffering a lower limb fracture and undergoing surgery. J Clin Nurs [Internet]. 2014 [cited 2018 jul 17]; 23(1-2):191-200. Available from: https://www.ncbi.nlm.nih.gov/ pubmed/23875652

21. Forsberg A, Vikman I, Wälivaara B-M, Engström A. Patients' perceptions of their postoperative recovery for one month. J Clin Nurs [Internet]. 2015 [cited 2018 oct 17]; 24 (13-14):1825-36. Available from: https://onlinelibrary.wiley.com/ doi/abs/10.1111/jocn.12793

22. Meirelles RN, Menezes DS, Luedy AR, Ribeiro H da C. Implantação de um Programa de Educação do Paciente em um Hospital Público. Revista Baiana de Saúde Pública [Internet]. 2015 [citado 2018 out 20]; 39(3):668-80. Disponível em: http://rbsp. sesab.ba.gov.br/index.php/rbsp/article/view/1812

23. Camargo PF, André LD, Lamari NM. Orientaçóes em saúde no processo de alta hospitalar em usuários reinternados do sistema único de saúde. Arquivos de Ciências da Saúde [Internet]. 2016 [citado 2018 set 14]; 23(3):38-43. Disponível em: http://www. cienciasdasaude.famerp.br/index.php/racs/article/ view/335

24. Bezerra MLR, Faria R de PR, Costa de Jesus CA, Dos Reis PED, Pinho DLM, Kamada I. Aplicabilidade da teoria do déficit do autocuidado de ordem no Brasil: uma revisão integrativa. J Manag Prim Health Care [Internet]. 2019 [citado 2019 dez 18]; 9: e16. Disponível em: http://www.jmphc.com.br/ jmphc/article/view/538

25. Stadler DV, Giordani AT, Ezaias Paulino GM, Sonobe HM, Zanardo RR, Valério MA. Estratégias para o ensino do autocuidado de pacientes cirúrgicos: uma revisão integrativa da literatura. Rev G\&S [Internet]. 2019 [citado 2019 dez 18];
00:128-41. Disponível em: https://periodicos.unb. br/index.php/rgs/article/view/23332

26. Tavares M de M, Gomes AMT, Barbosa DJ, Da Rocha JCCC, Bernardes MMR, Thiengo PC da $S$. Espiritualidade e religiosidade no cotidiano da enfermagem hospitalar. Revista de Enfermagem UFPE on line [Internet]. 2018 [citado 2018 oct 21]; 12(4): 1097-1102. Disponível em: https:// periodicos.ufpe.br/revistas/revistaenfermagem/ article/view/234780

27. Martins KP, Costa KN de FM, de Oliveira DST de, Valdevino SC, Rezende LCM, Da Costa TF. Atuaçáo do enfermeiro no preparo para a alta hospitalar de pacientes cirúrgicos. Rev. Pesqui. (Univ. Fed. Estado Rio J., Online) [Internet]. 2015 [citado 2018 set 06]; 7(1):1756-64. Disponível em: https://pesquisa.bvsalud.org/portal/resource/ $\mathrm{pt} / \mathrm{bde}-26689$

28. Nicolato FV, Santos CM, Castro EAB. Autocuidado e vivências do envelhecer de cuidadores familiares de idosos: contribuiçôes para enfermagem gerontológica. Tempus Actas de Saúde Coletiva [Internet]. 2017 [citado 2018 out 14]; 11(1):16986. Disponível em: https://pesquisa.bvsalud.org/ portal/resource/pt/biblio-880728

29. Souza KMJ de, Seixas CT, David HMSL, Costa AQ da. Contributions of Public Health to nursing practice. Rev Bras Enferm [Internet]. 2017 [cited 2018 sep 29]; 70(3):543-49. Available from: http://www.scielo.br/scielo.php?script=sci_ arttext\&pid $=$ S0034-71672017000300543

30. Regis CG, Batista NA. The nurse in the area of population health: concepts and competencies. Rev Bras Enferm [Internet]. 2015 [cited 2018 sept 03]; 68(5):548-54. Available from: http:// www.scielo.br/pdf/reben/v68n5/en_0034-7167reben-68-05-0830.pdf

31. Galavote HS, Zandonade E, Garcia ACP, Freitas P de SS, Seidl H, Contarato PC, et al. O trabalho do enfermeiro na atenção primária à saúde. Esc Anna Nery [Internet]. 2016 [cited 2018 out 21]; 20(1):90-98. Disponível em: http://www.scielo. br/scielo.php?script=sci_abstract\&pid=\$141481452016000100090\&lng=en\&tlng=pt

32. Andrade AM, Silva KL, Seixas CT, Braga PP. Atuação do enfermeiro na atenção domiciliar: uma revisão integrativa da literatura. Rev Bras Enferm [Internet]. 2017 [citado 2018 set 03]; 70(1):21019. Disponível em: http://www.scielo.br/scielo. php?script=sci_arttext\&pid=S0034-71672017000 100210

33. Santos FDRP, Nunes SFL, Pascoal LM, Silva JO, Almeida RP. Educação em saúde para pacientes no pós-operatório de cirurgias torácicas e abdominais. Rev Ciênc Ext [Internet]. 2015 [citado 2018 
out 16]; 11(1):171-77. Disponível em: https:// repositorio.ufma.br/jspui/handle/123456789/926

34. Chaves E de CL, Iunes DH, Moura C de C, Carvalho LC, Silva AM, Carvalho EC de, et al. Anxiety and spirituality in university students: a cross-sectional study. Rev Bras Enferm [Internet]. 2015 [cited 2018 oct 18]; 68(3):504-09. Available from: http://www.scielo.br/scielo.php?pid=S0034$71672015000300504 \&$ script=sci_arttext\&tlng=en

35. Fernandes MA, Meneses RT de, Franco SLG, Silva JS, Feitosa CDA. Transtornos de ansiedade: vivências de usuários de um ambulatório especializado em saúde mental. Revista de Enfermagem UFPE on line [Internet]. 2017 [citado 2018 out 18]; 11(10):3836-44. Disponível em: https:// periodicos.ufpe.br/revistas/revistaenfermagem/ article/view/25366

36. Coriolano-Marinus MW de L, Pavan MI, Lima LS de, Bettencourt AR de C. Validation of educational material for hospital discharge of patients with prolonged domiciliary oxygen prescription. Esc Anna Nery [Internet]. 2014 [cited 2018 sep 25]; 18(2):284-89. Available from: http://www.scielo.br/scielo.php?pid=S1414$81452014000200284 \&$ script=sci_arttext\&tlng=en

37. Rigon E, Mendes M, Dalazen JVC, Santos CE dos, Kirschener M, Toniollo CL, et al. Plano de alta como estratégia para comunicaçáo efetiva na internação hospitalar. Braz J Surg Clin Res [Internet]. 2014 [citado 2018 set 05]; 7(1): 30-34. Disponível em: https://www.mastereditora.com. br/periodico/20140602_103331.pdf

38. Linn AC, Azzolin K, Souza EN de. Association between self-care and hospital readmissions of patients with heart failure. Rev Bras Enferm [Internet]. 2016 [cited 2018 oct 14]; 69(3):50006. Available from: http://www.scielo.br/scielo. php?script $=$ sci_arttext $\&$ pid $=S 0034-7167$ 2016000300500\&lng=en\&tlng=en

39. Espinosa Aranzales Á, Enríquez Guerrero C, Leiva Aranzalez F, López Arévalo M, Castañeda Rodríguez L. Construcción colectiva de un concepto de cuidado humanizado en enfermería. Cienc. enferm. [Internet]. 2015 [citado 15 sep 2018]; 21(2): 39-49. Disponible en: https://scielo.conicyt. $\mathrm{cl} /$ scielo.php?script=sci_arttext $\&$ pid $=$ S071795532015000200005\&tlng=es

40. Costa SRD da, Castro EAB de. Autocuidado do cuidador familiar de adultos ou idosos dependentes após a alta hospitalar. Rev Bras Enferm [Internet]. 2014 [citado 2018 out 15]; 67(6):979-86. Disponível em: http://www.scielo.br/scielo.php?script=sci_ arttext\&pid=S0034-71672014000600979 